\section{Audio-range frequency-counter subroutine for the Apple II}

\section{O. BOWKER \\ Department of Ophthalmology, University of Wisconsin Madison, Wisconsin 53706}

This paper describes a 6502 assembly language subroutine that counts the frequency of an oscillating signal in the range of $.5 \mathrm{~Hz}-15 \mathrm{kHz}$. This routine has applications in experiments in which subjects are required to adjust the frequency of a periodic visual or auditory stimulus and the experimenter would like to obtain a precise record of the frequency after each adjustment (cf. Bowker \& Mandler, 1981).

Description. The frequency-counter routine was developed on an Apple II microcomputer with a Mountain real-time clock ${ }^{1}$ and was designed to be called from a BASIC language program. In our particular application, the oscillating signals were produced by a Wavetek 136 signal generator and were fed through an amplifier circuit to one of the Apple II game controller inputs (Hex Location \$C063). When called from the BASIC language program, the frequency-count subroutine first examines one of the real-time clock locations (Hex Location $\$ \mathrm{COC} 2$ ) and does not proceed until the value of that location increments. It then counts the number of low-to-high voltage transitions occurring at the game controller input before the next change in the real-time clock location. Each increment in the clock location represents a 16 -sec integration interval; we are particularly interested in signals oscillating below $30 \mathrm{~Hz}$. To save time, the more rapidly changing clock locations can be examined at higher oscillatory frequencies.

Three 8-bit memory locations, including the microprocessor's $Y$ register, count the voltage transitions. Over a 16-sec integration interval, this would theoretically allow the routine to count signals as high as $1 \mathrm{MHz}$. This is limited in practice by the running speed of the 6502 microprocessor. The routine is capable of reliably counting the frequencies of signals oscillating at rates of up to $15 \mathrm{kHz}$. This is independent of the signal's waveform (square, sine, triangular). A subroutine available on the Apple II microcomputer (beginning at Hex Location \$E2F2) returns the values of the accumulator (high-order byte) and $\mathrm{Y}$ register (low-order byte) to the USR(X) function called from the BASIC language program. Since the frequency is encoded in 3 bytes, calls are required to two separate locations in the assembly language subroutine.

Availability. A listing of the 6502 assembly language subroutine is provided in the appendix along with the BASIC language program. Additional details of the frequency-counting procedure may be obtained from the author, care of the Department of Ophthalmology, Room 568, Waisman Center, University of Wisconsin, Madison, Wisconsin 53706.

\section{RDFERENCE}

Bowker, D. O., \& Mander R, M. B. Binocular flicker appears faster than monocular flicker. Journal of the Optical Society of America, 1981, 71, 496-498.

\section{NOTE}

1. Mountain Computer, Inc., 300 Harvey West Boulevard, Santa Cruz, California 95060.

Appendix

\begin{tabular}{|c|c|c|c|c|c|c|c|}
\hline \multicolumn{6}{|c|}{6502 Frequency-Count Subroutine } & \multirow{2}{*}{\multicolumn{2}{|c|}{ BASIC Language Main Routine }} \\
\hline \multirow{2}{*}{$\frac{\text { Location }}{94 \mathrm{E} 0-}$} & \multicolumn{3}{|c|}{ Program Hex Code } & \multicolumn{2}{|c|}{ Program Mnemonics } & & \\
\hline & A9 & 00 & & LDA & $\# \$ 00$ & 10 & REM FREQUENCY COUNT MAIN ROUTINE \\
\hline $94 \mathrm{E} 2-$ & $8 \mathrm{D}$ & 50 & 95 & STA & $\$ 9550$ & 20 & REM D.O. BOWKER $2 / 82$ \\
\hline $94 \mathrm{E} 5-$ & $8 \mathrm{D}$ & 51 & 95 & STA & $\$ 9551$ & 30 & REM SET UP JUMP INSTRUCT FOR USR(X) \\
\hline $94 \mathrm{E} 8-$ & $8 \mathrm{D}$ & 52 & 95 & STA & $\$ 9552$ & 40 & POKE 10,76 \\
\hline $94 \mathrm{~EB}-$ & A0 & 00 & & LDY & $\# \$ 00$ & 50 & POKE 11,224 \\
\hline $94 \mathrm{ED}-$ & $\mathrm{AE}$ & $\mathrm{C} 2$ & $\mathrm{CO}$ & LDX & $\$ \mathrm{COC} 2$ & 60 & POKE 12,148 \\
\hline $94 \mathrm{~F} 0-$ & EC & $\mathrm{C} 2$ & $\mathrm{CO}$ & CPX & $\$ \mathrm{COC} 2$ & 70 & REM CALL SUBROUTINE \\
\hline $94 F 3-$ & D0 & 03 & & BNE & $\$ 94 \mathrm{~F} 8$ & 80 & $F 1=\operatorname{USR}(0)$ \\
\hline $94 \mathrm{~F} 5-$ & $4 C$ & F0 & 94 & $\mathrm{JMP}$ & $\$ 94 \mathrm{FO}$ & 90 & REM ACCOUNT FOR ACCUM. SIGN BIT \\
\hline $94 \mathrm{~F} 8-$ & $\mathbf{A E}$ & $\mathrm{C} 2$ & $\mathrm{CO}$ & LDX & $\$ \mathrm{COC} 2$ & 100 & IF $F 1<0$ THEN F1 $=F 1+65536$ \\
\hline $94 \mathrm{FB}-$ & $\mathrm{EC}$ & $\mathrm{C} 2$ & $\mathrm{CO}$ & CPX & $\$ \mathrm{COC} 2$ & 110 & REM SET UP NEW JUMP LOCATION \\
\hline $94 \mathrm{FE}-$ & F0 & 07 & & $\mathrm{BEQ}$ & $\$ 9507$ & 120 & POKE 11,68 \\
\hline $9500-$ & $\mathrm{AD}$ & 50 & 95 & LDA & $\$ 9550$ & 130 & POKE 12,149 \\
\hline $9503-$ & 20 & F2 & E2 & JSR & $\$ E 2 F 2$ & 140 & REM CALL SUBROUTINE AGAIN \\
\hline $9506-$ & 60 & & & RTS & & 150 & $\mathrm{~F} 2=\mathrm{USR}(0)$ \\
\hline $9507-$ & $\mathrm{AD}$ & 63 & $\mathrm{CO}$ & LDA & $\$ C 063$ & 160 & REM COMPUTE FREQUENCY \\
\hline $950 \mathrm{~A}-$ & E9 & 80 & & SBC & $\# \$ 80$ & 170 & PRINT "FREQUENCY IS "; $(\mathrm{F} 1+(\mathrm{F} 2 * 65536)) / 16$ \\
\hline $950 \mathrm{C}-$ & 30 & 05 & & BMI & $\$ 9513$ & 180 & END \\
\hline
\end{tabular}


6502 Frequency-Count Subroutine Continued

\begin{tabular}{|c|c|c|c|c|c|}
\hline \multirow{2}{*}{$\frac{\text { Location }}{950 \mathrm{E}-}$} & \multicolumn{3}{|c|}{ Program Hex Code } & \multicolumn{2}{|c|}{ Program Mnemonics } \\
\hline & A9 & 01 & & LDA & $\# \$ 01$ \\
\hline $9510^{--}$ & $4 \mathrm{C}$ & $1 B$ & 95 & JMP & $\$ 951 \mathrm{~B}$ \\
\hline $9513-$ & A9 & 00 & & LDA & $\# \$ 00$ \\
\hline $9515-$ & 8D & $4 \mathrm{~F}$ & 95 & STA & $\$ 954 \mathrm{~F}$ \\
\hline $9518-$ & $4 \mathrm{C}$ & FB & 94 & JMP & $\$ 94 \mathrm{FB}$ \\
\hline $951 \mathrm{~B}-$ & CD & $4 \mathrm{~F}$ & 95 & CMP & $\$ 954 \mathrm{~F}$ \\
\hline $951 \mathrm{E}-$ & Do & 03 & & BNE & $\$ 9523$ \\
\hline $9520-$ & $4 C$ & FB & 94 & JMP & $\$ 94 \mathrm{FB}$ \\
\hline $9523-$ & $8 D$ & $4 \mathrm{~F}$ & 95 & STA & $\$ 954 \mathrm{~F}$ \\
\hline $9526-$ & $\mathrm{CO}$ & $\mathrm{FF}$ & & CPY & $\# \$ F F$ \\
\hline $9528-$ & D0 & 15 & & $\mathrm{BNE}$ & $\$ 953 \mathrm{~F}$ \\
\hline $952 \mathrm{~A}-$ & A9 & FF & & LDA & $\# \$ F F$ \\
\hline $952 \mathrm{C}-$ & $\mathrm{CD}$ & 50 & 95 & CMP & $\$ 9550$ \\
\hline $952 \mathrm{~F}-$ & D0 & $0 \mathrm{~B}$ & & $\mathrm{BNE}$ & $\$ 953 \mathrm{C}$ \\
\hline $9531-$ & $\mathrm{CD}$ & 51 & 95 & CMP & $\$ 9551$ \\
\hline $9534-$ & DO & 03 & & BNE & $\$ 9539$ \\
\hline $9536-$ & $\mathrm{EE}$ & 52 & 95 & INC & $\$ 9552$ \\
\hline $9539-$ & EE & 51 & 95 & INC & $\$ 9551$ \\
\hline $953 \mathrm{C}-$ & $\mathrm{EE}$ & 50 & 95 & INC & $\$ 9550$ \\
\hline $953 \mathrm{~F}-$ & $\mathrm{C} 8$ & & & INY & \\
\hline $9540-$ & $4 C$ & FB & 94 & JMP & $\$ 94 F B$ \\
\hline $9543-$ & 00 & & & BRK & \\
\hline $9544-$ & $\mathrm{AC}$ & 51 & 95 & LDY & $\$ 9551$ \\
\hline $9547-$ & $\mathrm{AD}$ & 52 & 95 & LDA & $\$ 9552$ \\
\hline $954 \mathrm{~A}-$ & 20 & $\mathrm{~F} 2$ & $\mathrm{E} 2$ & JSR & $\$ E 2 F 2$ \\
\hline 954D- & 60 & & & RTS & \\
\hline $954 \mathrm{E}-$ & 00 & & & BRK & \\
\hline $954 \mathrm{~F}-$ & 00 & & & BRK( & ESERVED) \\
\hline $9550-$ & 00 & & & BRK & ESERVED) \\
\hline $9551-$ & 00 & & & BRK & ESER VED) \\
\hline $9552-$ & 00 & & & BRK & ESERVED) \\
\hline
\end{tabular}

(Accepted for publication February 23, 1982.) 